\title{
Anatomy of Starbursts in Extragalactic Giant HII Regions: M51 Case Study
}

\author{
Jessica Marie Evans ${ }^{1}$ and You-Hua $\mathrm{Chu}^{2}$ \\ ${ }^{1,2}$ Department of Astronomy, University of Illinois \\ 1002 W. Green St, Urbana, IL, United States \\ 1 email: evans35@illinois.edu \\ ${ }^{2}$ email: yhchu@illinois .edu
}

\begin{abstract}
Extragalactic giant HII regions (EGHRs) are sites of active, concentrated star formation, and thus provide excellent labs to analyze the starburst phenomenon. Although they have been known for a long time, ground-based observations cannot resolve the physical structures and stellar content of EGHRs. The high resolution and sensitivity of Hubble Space Telescope (HST) are ideal for detailed studies of EGHRs. We have searched the Hubble Legacy Archives (HLA) and found 17 nearby galaxies, within $\sim 15 \mathrm{Mpc}$, with $\mathrm{H} \alpha$ and continuum images; to determine the best methods for analyzing these data, we perform an in-depth analysis of the EGHRs in M51. M51 is a face-on spiral galaxy $\sim 8.4 \mathrm{Mpc}$ away, with well-resolved multi-wavelength observations in the HLA. We sample the 25 most luminous HII regions in M51, many of which are bonafide EGHRs with an $\mathrm{H}$-alpha luminosity $>10^{39} \mathrm{ergs} \mathrm{s}^{-1}$. We use the $\mathrm{H} \alpha$ image to study the distribution and physical structure of the gas in each HII region and determine its $\mathrm{H} \alpha$ luminosity and required ionizing flux. We use the continuum images to determine whether super stellar clusters (SSCs) are found in these HII regions, and use photometric measurements to determine the mass and age spread of the resolved stellar population. These are then compared with the interstellar structures. The results help us provide the groundwork for studying EGHRs in multiple galaxies and elucidate the starburst phenomenon by investigating questions such as: What role does environment play in the formation of EGHRs? How do EGHRs evolve? How does star formation proceed in an EGHR?
\end{abstract}

Keywords. HII regions - galaxies: star clusters
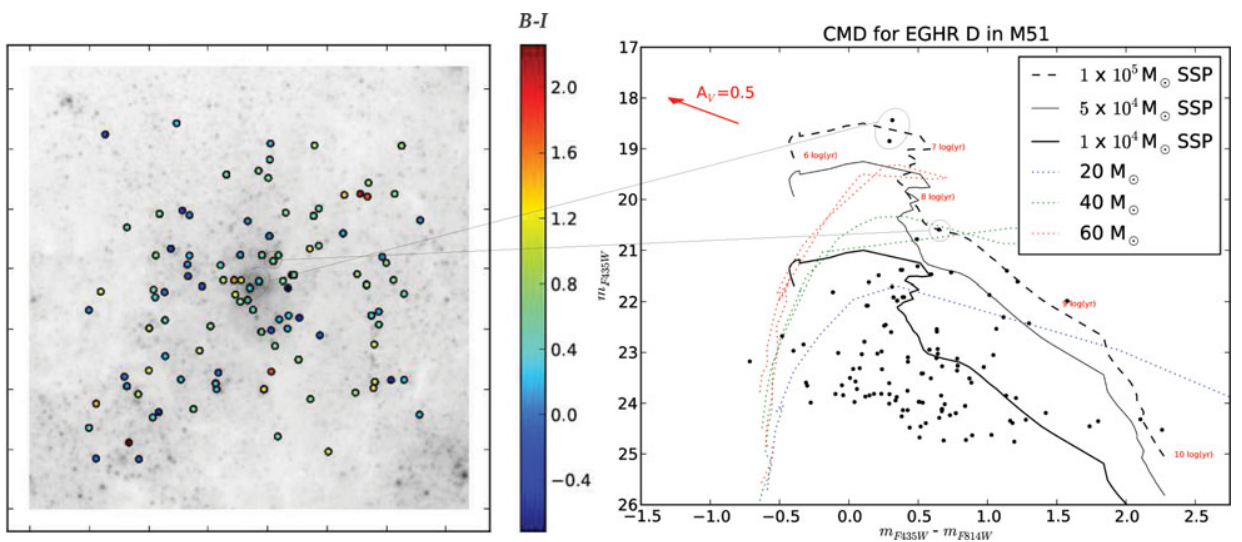

Figure 1. Blue continuum (F435W) image of third brightest EGHR in M51 with overplots of $B-I$ colors of the stellar content (left) with the corresponding $B$ vs. $B-I$ color-magnitude diagram. The brightest detections reside along the $10^{5} \mathrm{M}_{\odot}$ single stellar population evolutionary track. 